\title{
Pre-Main Sequence Stars seen by ISO*
}

\author{
Dario Lorenzetti (dloren@mporzio.astro.it) \\ INAF - Osservatorio Astronomico di Roma - Via Frascati 33 - 00040 Monte \\ Porzio (Roma) - Italy
}

\begin{abstract}
Observations of pre-main sequence objects (T Tauri, Herbig Ae/Be and FU Orionis stars) obtained with the instrumentation on board the Infrared Space Observatory (ISO) are reviewed. All the observations have been mainly carried out by using the two spectrographs SWS and LWS, adopting their low resolution modes and such data have been used both for lines detection and to reconstruct the spectral energy distributions. Line emission and photometric behaviour of pre-main sequence objects have been analyzed in the framework of the current models, discussing the agreement (or disagreement) with them and trying to derive the questions which should be answered by the forthcoming FIR instrumentation.
\end{abstract}

Keywords: Pre-main sequence, circumstellar matter, ISO, Infrared spectroscopy

Received: 7 July 2004

\section{Introduction}

The Infrared Space Observatory (ISO) (Kessler et al., 1996; Kessler et al., 2003) has been the first facility outside the atmosphere able to provide, through its instrumental capabilities, a systematic diagnosis of the phenomena (photodissociation, shocks, PAH and dust emission) occurring in the close environment of the pre-main sequence stars. In this area of astrophysical interests, the importance of the spectroscopical approach has largely exceeded that of the photometric one: indeed, the IRAS survey had already made possible the first evaluation of the amount of energy emitted by these systems, not allowing, however, any investigation of the mechanisms responsible for the gas excitation. Despite its peculiar nature of Observatory dedicated to selected targets, ISO has provided, in the specific topic of the pre-main sequence, also complete surveys of significant samples, whose unbiased nature has revealed as crucial, also representing a useful starting point for the FIR/sub-mm facilities of the next future.

For reason of compactness, the present review will account only for published papers based on ISO data on this topic (37 during the period

* Based on observations with ISO, an ESA project with instruments funded by ESA Member States (especially the PI countries: France, Germany, the Netherlands and the United Kingdom) and with the participation of ISAS and NASA.

(C) Manuscript - To be published by Springer in 2005.

Space Science Review ISO Special Issue. 
1996 - beginning of 2004), although interesting information can be also retrieved from more than 50 conference proceedings and about $10 \mathrm{PhD}$ theses dealing essentially with ISO studies on Herbig Ae/Be stars. The reader is also referred to the excellent review by van Dishoeck (2004) which gives a complete analysis of the ISO spectroscopic results.

\section{T Tauri and Herbig Ae/Be stars}

\subsection{ISO OBSERVATIONS}

Data from the spectrometers on board ISO - mainly SWS (de Graauw et al., 1996; Leech et al., 2003) and LWS (Clegg et al., 1996; Gry et al., 2003) and PHOT-S (Lemke et al., 1996; Laureijs et al., 2003) to a lesser extent, allowed to obtain low resolution $(\mathcal{R} \sim 200-1000)$ spectra between 3 and $200 \mu \mathrm{m}$ for a significant samples of T Tauri (TTS) and Herbig AeBe (HAEBE) stars. Due to the intrinsic faintness of its members, the former class has been less investigated than the latter, and all the objects observed with ISO are listed in the first six columns of Table I, along with an indication of the papers referring to them. The binary system formed by $\mathrm{T}$ Tau ( $\mathrm{N}$ and $\mathrm{S}$ ) has been revealed as the pre-main sequence object richest of atomic and molecular lines (van den Ancker et al. 1999; Spinoglio et al. 2000). Two surveys, the one on pure rotational $\mathrm{H}_{2}$ lines (Thi et al. 1999, 2001), and the second on silicate features (Natta et al. 2000) have obtained significant results discussed in the following sections.

Concerning the HAEBE, a complete compilation of SWS and LWS data has been recently presented by Elia et al. (2004) who searched the ISO mission data bank for the 108 bona fide HAEBE stars listed in the catalog of Thé et al. (1994, their Table 1) obtaining 36 objects with at least one SWS or LWS observation of good quality. More recently, Acke \& van den Ancker (2004), in their study of disks around 46 HAEBE, have studied all the ISO spectra obtained with different facilities, namely SWS, ISOPHOT-S and ISOCAM (Cesarsky et al., 1996; Blommaert et al., 2003) CVF scans. Other ISOCAM CVF scans of 8 nearby HAEBE have been presented by Siebenmorgen et al. (2000). To have a complete census of the available ISO data concerning the HAEBE, spectrophotometric data have been complemented with far IR ISOPHOT photometry (obtained with ten broad band filters from 4.8 to $200 \mu \mathrm{m}$, partially overlapping the IRAS ones) of $7 \mathrm{HAEBE}$ (Abraham et al. 2000). All the investigated HAEBE are listed in Table II. 
Table I. ISO Observations of T Tauri and FU Orionis stars.

\begin{tabular}{|c|c|c|c|c||c|c|c|}
\hline TTS & SWS & LWS & TTS & PHOT-S & FU Ori & SWS & LWS \\
\hline AA Tau & 1 & 2,4 & CT Cha & 7 & RNO 1B & & 3,9 \\
DM Tau & 1 & & Glass I & 7 & Z CMa $^{a}$ & 10 & 3,9 \\
DR Tau & 1 & & LkH $\alpha 332-20$ & 7 & V346 Nor & & 3,9 \\
GG Tau & 1,8 & 4 & SX Cha & 7 & Re $13^{b}$ & & 3,9 \\
GO Tau & 1 & & VW Cha & 7 & V1057 Cyg & & 9 \\
RY Tau & 1 & & VZ Cha & 7 & V1331 Cyg & & 9 \\
T Tau & 5 & 3,6 & WX Cha & 7 & V1735 Cyg & & 3,9 \\
DG Tau & & 3 & WW Cha & 7 & & \\
HL Tau & & 3 & XX Cha & 7 & & \\
GM Aur & 1 & & & & & & \\
LkCa 15 & 1 & 2 & & & & & \\
\hline
\end{tabular}

Notes to the Table:

a - Considered also as a HAEBE (see Table II).

b - It is not a FU Ori. It lies in proximity of V346 Nor (less than 1 arcmin).

- References: 1. Thi et al. 2001; 2. Chiang et al. 2001; 3. Pezzuto et al. 2002; 4. Creech-Eakman et al. 2002; 5. van den Ancker et al. 1999; 6. Spinoglio et al.; 7. Natta et al. 2000; 8. Thi et al. 1999; 9. Lorenzetti et al. 2000; 10. Acke \& van den Ancker (2004).

\subsection{The SPECTRAL ENERGY DistribUtions}

Low resolution ISO spectra (3-200 $\mu \mathrm{m})$ have been extensively used, also in combination with literature data sets (UV, optical, near IR, sub-mm, radio) to construct accurate spectral energy distributions (SEDs). Only 3 TTS (namely AA Tau, GG Tau and LkCa 15) have been investigated in this sense, and the results are incorporated in the following discussion dealing with HAEBE. For these latter, the dust continuum behaves very differently from source to source: some objects present their SED rising with the wavelength (up to about $100 \mu \mathrm{m}$ ), other rather flat or even declining (see Figure 1), confirming the fact that HAEBE do not constitute a homogeneous class of object, at variance with the T Tauri class.

ISO data have played a major role in creating some convergence on the long-standing debate about the spatial distribution (spherical 
Table II. ISO Observations of HAEBE.

\begin{tabular}{|c|c|c|c|c|}
\hline Source & SWS & LWS & CAM & PHOT \\
\hline $\mathrm{LkH} \alpha 198$ & & $1,5,27$ & & 3 \\
\hline V376 Cas & 1 & $1,5,27$ & & 3 \\
\hline VX Cas & & & & 2 \\
\hline Elias 3-1 & $1,2,26,30$ & & & \\
\hline $\mathrm{AB}$ Aur & $1,2,8,12,13,14,15,30$ & $1,9,13$ & & \\
\hline MWC 480 & $1,2,30$ & $1,9,11,28$ & & \\
\hline UX Ori & 30,31 & & & 2 \\
\hline HD 34282 & 1,2 & 1,28 & & \\
\hline HD $34700^{a}$ & 2 & & & 2 \\
\hline HD $35187^{a}$ & 2 & & & \\
\hline HD 36112 & 30 & $1,9,11,28$ & & \\
\hline CQ Tau & 30 & $1,9,10,11,28$ & & \\
\hline BF Ori & & & & 2 \\
\hline RR Tau & & & & 2 \\
\hline MWC 137 & 1 & 1,28 & & \\
\hline $\mathrm{Z} \mathrm{CMa}^{b}$ & 1,2 & 1,27 & & \\
\hline He 3-554 & 2 & & & 2 \\
\hline HD 97048 & $1,2,26,32$ & $1,5,27$ & 2,4 & 2 \\
\hline HD $97300^{a}$ & & & 4,20 & 7 \\
\hline HD $100453^{a}$ & $2,8,12,15$ & & & 2 \\
\hline HD 100546 & $1,2,8,12,15,17,29,32$ & $1,17,28$ & & 2 \\
\hline HD 104237 & $1,2,8,12,15,31$ & 1,28 & & 2 \\
\hline IRAS 12496-7650 & 1,2 & $1,5,6,18$ & & 2 \\
\hline HD $135344^{a}$ & $2,8,12,15$ & & & 2 \\
\hline HD $139614^{a}$ & $2,8,12,15$ & & & 2 \\
\hline HD 141569 & 1,2 & 1,28 & & 2 \\
\hline HD $142527^{a}$ & $2,8,12,15,22$ & 22 & & 2 \\
\hline HD 142666 & $1,2,8,12,15,31$ & 1,28 & & 2 \\
\hline HD 144432 & $1,2,8,12,15,31$ & 1,28 & & 2 \\
\hline HR 5999 & 1,2 & & 2,4 & 2 \\
\hline $\operatorname{HR} 6000^{a}$ & & & 4 & \\
\hline He $3-1191$ & 1,2 & & & \\
\hline HD 150193 & $1,2,8,12,15,30,31$ & & & 2 \\
\hline $\mathrm{AK} \mathrm{Sco}^{a}$ & 2 & & & 2 \\
\hline $\mathrm{CoD}-42^{\circ} 11721$ & $1,2,21,32$ & $1,5,27$ & & 2 \\
\hline KK Oph & 32 & & & \\
\hline HD 163296 & $1,2,8,12,13,14,15,30,31$ & & & 2 \\
\hline HD $169142^{a}$ & $2,8,12,15$ & & & 2 \\
\hline MWC 297 & $1,2,25$ & $1,5,25,27$ & & \\
\hline VV Ser & & & & 2 \\
\hline MWC 300 & 1 & & & \\
\hline TY CrA & 1 & 1,28 & 4 & \\
\hline $\mathrm{R} \mathrm{CrA}$ & 1,2 & $1,5,6,27$ & & 2 \\
\hline $\mathrm{T} \mathrm{CrA}^{a}$ & 2 & 27 & & 2 \\
\hline
\end{tabular}


Table II. continued

\begin{tabular}{|c|c|c|c|c|}
\hline Source & SWS & LWS & CAM & PHOT \\
\hline HD 179218 & $1,2,8,12,15,32$ & & 2,4 & \\
HD 176386 & & & 4 & \\
WW Vul & $1,2,30$ & & & 2,10 \\
BD +40 4124 & $1,2,19,24,32$ & $1,9,24,28$ & & 2 \\
LkH $\alpha 224$ & $1,2,24$ & 1,24 & & \\
LkH $\alpha 225^{a}$ & 2,24 & 24 & & \\
PV Cep & 1,2 & $1,5,27$ & & 2,3 \\
HD 200775 & $1,2,16,23$ & $1,16,28$ & 2 & \\
V645 Cyg & 1,2 & $1,5,27$ & & \\
BD +65 ${ }^{\circ} 637$ & & & & 3 \\
BD +46 3471 & & & & \\
SV Cep & 2 & $1,5,6,27$ & & 3 \\
LkH $\alpha 234$ & & $1,9,28$ & & 3 \\
LkH $\alpha 233$ & & & & 3 \\
MWC 1080 & $1,2,21$ & 1,27 & & \\
\hline
\end{tabular}

Notes to the Table:

a - Not listed in the Thé et al. Table 1.

b - Considered also as a FU Ori object (see Table I).

- References: 1. Elia et al. 2004; 2. Acke \& van den Ancker (2004); 3. Abraham et al. 2000; 4. Siebenmorgen et al. 2000; 5. Lorenzetti et al. 1999; 6. Giannini et al. 1999; 7. Gürtler et al. 1999.; 8. Dominik et al. 2003; 9. Creech-Eakman et al. 2002; 10. Natta et al. 2001; 11. Chiang et al. 2001; 12. Meeus et al. 2001; 13. Bouwman et al. 2000; 14. van den Ancker et al. 2000a; 15. Bouwman et al. 2001; 16. Fuente et al. 2000; 17. Malfait et al. 1998; 18. Nisini et al. 1996; 19: Wesselius et al. 1996; 20. Siebenmorgen et al. 1998; 21. Benedettini et al. 1998; 22. Malfait et al. 1999; 23. Fuente et al. 1999; 24. van den Ancker et al. 2000b; 25. Benedettini et al. 2001; 26. van Kerckhoven et al. 2002; 27. Pezzuto et al. 2002; 28. Lorenzetti et al. 2002; 29. Bouwman et al. 2003; 30. Thi et al. 2001; 31. van Boekel R. et al. 2003; 32. Peeters et al. 2002.

envelopes vs. flattened structures) of the circumstellar matter around HAEBE. The reason for that relies on the fact they represent a common starting point for different authors for evaluating the capabilities of the different models, namely a data-base which covers, at spectrophotometric resolution, a rather wide spectral range. Nevertheless we have to remember that the ISO spatial resolution is relatively poor, therefore 
Table III. Emission lines observed in Pre-Main Sequence objects.

\begin{tabular}{|c|c|c|}
\hline $\begin{array}{l}\text { wavelength } \\
\qquad(\mu \mathrm{m})\end{array}$ & Identification & Sources \\
\hline $2.4-7.5$ & $\begin{array}{l}\mathrm{H}: \mathrm{Br}, \mathrm{Pf}, \mathrm{Hu} \\
\text { Series }\end{array}$ & $\begin{array}{c}\mathrm{CoD}-42^{\circ} 11721(6), \mathrm{MWC} 297(7), \mathrm{BD}+40^{\circ} 4124(9), \\
\mathrm{HD} 200775(10), \mathrm{MWC} 1080(6),\end{array}$ \\
\hline 6.91 & $\mathrm{H}_{2} \mathrm{~S}(5)$ & $\mathrm{BD}+40^{\circ} 4124(9), \mathrm{LkH} \alpha 224(9,11), \mathrm{HD} 200775(10)$ \\
\hline 8.02 & $\mathrm{H}_{2} \mathrm{~S}(4)$ & $\mathrm{BD}+40^{\circ} 4124(9,11), \mathrm{LkH} \alpha 224(9,11), \mathrm{HD} 200775(10)$ \\
\hline 9.66 & $\mathrm{H}_{2} \mathrm{~S}(3)$ & $\mathrm{BD}+40^{\circ} 4124(9,11), \mathrm{LkH} \alpha 224(9,11), \mathrm{HD} 200775(10)$ \\
\hline 12.28 & $\mathrm{H}_{2} \mathrm{~S}(2)$ & $\mathrm{BD}+40^{\circ} 4124(9,11), \mathrm{LkH} \alpha 224(9,11), \mathrm{HD} 200775(10)$ \\
\hline 17.03 & $\mathrm{H}_{2} \mathrm{~S}(1)$ & $\begin{array}{l}\text { GG Tau (13), AB Aur (1), MWC } 480(1), \operatorname{HD} 36112(1) \\
\text { CQ Tau (1), HD } 150193(1), \operatorname{HD} 163296(1) \\
\text { BD }+40^{\circ} 4124(9,11), \operatorname{LkH} \alpha 224(9,11), \operatorname{HD} 200775(10)\end{array}$ \\
\hline 18.71 & {$[\mathrm{SIII}]{ }^{3} \mathrm{P}_{2^{-}}{ }^{3} \mathrm{P}_{1}$} & $\mathrm{CoD}-42^{\circ} 11721(2)$ \\
\hline 25.25 & {$[\mathrm{SI}]{ }^{3} \mathrm{P}_{1-}{ }^{3} \mathrm{P}_{2}$} & $\mathrm{LkH} \alpha 224(9)$ \\
\hline 25.99 & {$[\mathrm{FeII}]{ }^{6} \mathrm{D}_{7 / 2^{-}}{ }^{6} \mathrm{D}_{9 / 2}$} & $\mathrm{BD}+40^{\circ} 4124(9)$ \\
\hline 28.22 & $\mathrm{H}_{2} \mathrm{~S}(0)$ & $\begin{array}{c}\text { GG Tau (13), AB Aur (1),UX Ori (1), CQ Tau (1), } \\
\text { HD } 150193 \text { (1), HD163296 (1) }\end{array}$ \\
\hline 33.48 & {$[\mathrm{SIII}]{ }^{3} \mathrm{P}_{1-}{ }^{3} \mathrm{P}_{0}$} & $\mathrm{CoD}-42^{\circ} 11721(2)$ \\
\hline 34.81 & {$[\mathrm{SiII}]{ }^{2} \mathrm{P}_{3 / 2^{-}}{ }^{2} \mathrm{P}_{1 / 2}$} & $\begin{array}{c}\mathrm{CoD}-42^{\circ} 11721(2), \mathrm{BD}+40^{\circ} 4124(9) \\
\mathrm{LkH} \alpha 224(9), \mathrm{HD} 200775(10)\end{array}$ \\
\hline 51.81 & {$[\mathrm{OIII}]{ }^{3} \mathrm{P}_{2-}{ }^{3} \mathrm{P}_{1}$} & V1057 Cyg (12), BD+40² $4124(4,8,9)$ \\
\hline 57.33 & {$[\mathrm{NIII}]{ }^{2} \mathrm{P}_{3 / 2^{-}}{ }^{2} \mathrm{P}_{1 / 2}$} & V1057 Cyg (12) \\
\hline 63.18 & {$[\mathrm{OI}]{ }^{3} \mathrm{P}_{1-}{ }^{3} \mathrm{P}_{2}$} & $\begin{array}{c}\text { RNO1B (12), ZCMa (12), V346 Nor (12), } \\
\text { Re13 (12), V1057 Cyg (12), V1331 Cyg (12), } \\
\text { V1735 Cyg (12), LkH } \alpha 198(2), \text { V376 Cas }(2), \\
\text { AB Aur (4,8), CQ Tau (8), MWC } 137(4), \operatorname{HD} 97048(2), \\
\text { HD100546 (4), HD104237 (4), IRAS } 12496(2,5), \\
\text { CoD-42 }{ }^{\circ} 11721(2), \text { MWC } 297(2), \text { TY CrA }(4), \text { R CrA (2), } \\
\text { BD }+40^{\circ} 4124(4,8,9), \text { PV Cep (2), HD200775 (4,10), } \\
\text { V645 Cyg }(2), \operatorname{LkH} \alpha 234(2), \operatorname{LkH} \alpha 233(4,8), \text { MWC } 1080(2)\end{array}$ \\
\hline 65.69 & CO 40-39 & $\mathrm{R}$ CrA (2) \\
\hline 71.19 & $\mathrm{OH}^{2} \Pi_{1 / 2,7 / 2^{-}}{ }^{2} \Pi_{1 / 2,5 / 2}$ & $\mathrm{R}$ CrA (3) \\
\hline 79.15 & $\mathrm{OH}^{2} \Pi_{1 / 2,1 / 2^{-}}{ }^{2} \Pi_{3 / 2,3 / 2}$ & Z CMa (12), R CrA $(2,3)$, LkH $\alpha 234$ (3) \\
\hline 84.51 & $\mathrm{OH}^{2} \Pi_{3 / 2,7 / 2^{-}}{ }^{2} \Pi_{3 / 2,5 / 2}$ & V1057 Cyg (12), R CrA $(2,3)$, LkH $\alpha 234$ (3) \\
\hline 88.36 & {$[\mathrm{OIII}]{ }^{3} \mathrm{P}_{1-}{ }^{3} \mathrm{P}_{0}$} & $\begin{array}{c}\text { V1057 Cyg (12), V1331 Cyg (12) } \\
\text { CoD }-42^{\circ} 11721(2), \mathrm{BD}+40^{\circ} 4124(4,8,9), \text { V645 Cyg }(2)\end{array}$ \\
\hline
\end{tabular}


Table III. continued

\begin{tabular}{|c|c|c|}
\hline $\begin{array}{l}\text { wavelength } \\
\qquad(\mu \mathrm{m})\end{array}$ & Identification & Sources \\
\hline 121.80 & {$[\mathrm{NII}]{ }^{3} \mathrm{P}_{2-}{ }^{3} \mathrm{P}_{1}$} & $\begin{array}{l}\text { V346 Nor (12), V1057 Cyg (12), V1331 Cyg (12), } \\
\text { V1735 Cyg (12), CoD }-42^{\circ} 11721(2), \operatorname{BD}+40^{\circ} 4124(4,8)\end{array}$ \\
\hline 124.19 & CO 21-20 & $\mathrm{R} \mathrm{CrA}(2)$ \\
\hline 130.37 & CO 20-19 & IRAS $12496(2)$ \\
\hline 137.20 & CO $19-18$ & IRAS $12496(2,3), \mathrm{R}$ CrA $(2,3), \mathrm{LkH} \alpha 234(3)$ \\
\hline 144.78 & CO $18-17$ & IRAS $12496(2,3), \mathrm{LkH} \alpha 234(3)$ \\
\hline 145.53 & {$[\mathrm{OI}]{ }^{3} \mathrm{P}_{0^{-}}{ }^{3} \mathrm{P}_{1}$} & $\begin{array}{l}\text { RNO1B (12), Z CMa (12), V346 Nor (12), V1331 Cyg (12), } \\
\text { V1735 Cyg (12), MWC } 137(4), \operatorname{HD} 100546(4), \operatorname{IRAS} 12496(2,5), \\
\text { CoD- } 42^{\circ} 11721(2), \text { MWC } 297(2), \text { TY CrA }(4), \\
\text { BD }+40^{\circ} 4124(4,8,9), \operatorname{HD} 200775(4,10), \operatorname{LkH} \alpha 234(2), \\
\operatorname{LkH} \alpha 233(4,8), \text { MWC } 1080(2)\end{array}$ \\
\hline 153.27 & CO $17-16$ & $\begin{array}{l}\text { V1057 Cyg (12), IRAS } 12496(2,3,5), \text { TY CrA }(4) \\
\quad \text { R CrA }(2,3), \operatorname{BD}+40^{\circ} 4124(4,9), \operatorname{LkH} \alpha 234(2,3)\end{array}$ \\
\hline 157.68 & {$[\mathrm{CII}]{ }^{2} \mathrm{P}_{3 / 2^{-}}{ }^{2} \mathrm{P}_{1 / 2}$} & 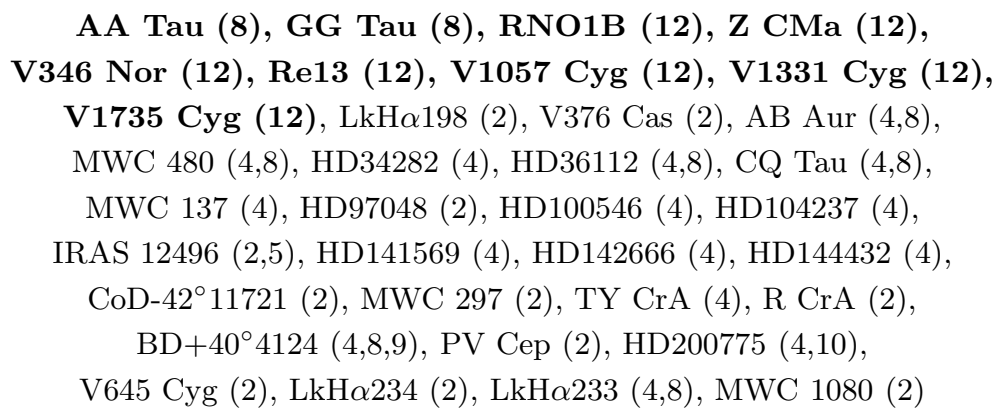 \\
\hline 162.81 & $\mathrm{CO} 16-15$ & $\begin{array}{l}\text { Re13 (12), V1057 Cyg (12), IRAS } 12496(3,5), \text { TY CrA (4), } \\
\text { R CrA }(2,3), \text { BD }+40^{\circ} 4124(4,9), \operatorname{HD} 200775(4), \operatorname{LkH} \alpha 234(2,3)\end{array}$ \\
\hline 173.63 & $\mathrm{CO} 15-14$ & IRAS $12496(3,5), \mathrm{R}$ CrA $(2,3), \mathrm{BD}+40^{\circ} 4124(4,9), \mathrm{LkH} \alpha 234(2,3)$ \\
\hline 179.53 & $\mathrm{o}-\mathrm{H}_{2} \mathrm{O} 22_{12}-1_{01}$ & $\mathrm{BD}+40^{\circ} 4124(4)$ \\
\hline 186.00 & CO $14-13$ & IRAS $12496(3), \mathrm{R}$ CrA $(2,3), \mathrm{BD}+40^{\circ} 4124(4,9), \mathrm{LkH} \alpha 234(2,3)$ \\
\hline
\end{tabular}

Notes to the Table:

- T Tauri and FU Orionis stars are given in boldface. The remaining refer to HAEBE stars. An exception is done for T Tauri itself, whose spectrum presents a plenty of $\mathrm{CO}, \mathrm{OH}, \mathrm{H}_{2} \mathrm{O}$ and atomic lines which are not indicated in the present Table (see Figure 4).

- References: 1. Thi et al. 2001; 2. Lorenzetti et al. 1999; 3. Giannini et al. 1999; 4. Lorenzetti et al. 2002; 5. Nisini et al. 1996; 6. Benedettini et al. $1998 ; 7$. Benedettini et al. 2001; 8. Creech-Eakman et al. 2002; 9. van den Ancker et al. 2000b; 10. Fuente et al. 2000; 11. Wesselius et al. 1996; 12. Lorenzetti et al. 2000; 13. Thi et al. 1999. 

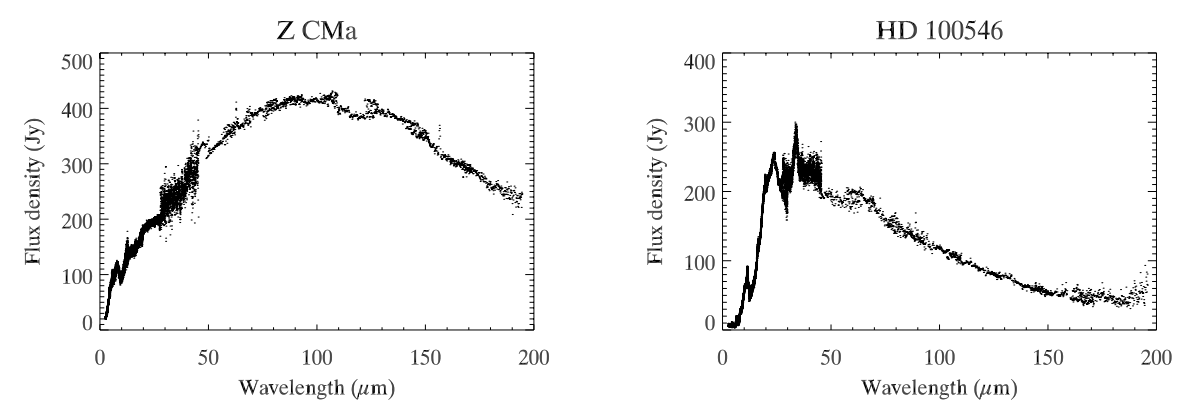

Figure 1. ISO spectra of two HAEBE, as obtained after accurate reduction of the spectrometer observations, showing two substantially different SEDs.

ISO results have to be treated with a certain care and need sometime a further confirmation.

The SWS data from 14 isolated Herbig stars with A-F spectral types, have been firstly presented by Meeus et al. (2001), who defined two key groups based upon the spectral shape of the infrared region. The first group contains sources for which the continuum can be reconstructed by a power law (optically thick, geometrically thin disk) and by a black body at about $200 \mathrm{~K}$ (flare region); the second group sources only need a power law to fit their continuum. The presence or not of a solid state bands contribution furtherly subdivides both groups. They explain the observed SEDs of the second group by assuming that the inner part of the disk is partially optically thick, shielding the outer parts from direct stellar radiation, which prevents the disk from flaring, therefore the black body component is not observed. They also conclude that evidence exists for grain growth in the HAEBE disks: the first group sources may evolve into the second one which has evidence for larger grains and lacks of flaring.

The same 14 HAe have been also considered by Dominik et al. (2003), who using the Dullemond et al. (2001) model, find that the different spectra can be interpreted as pure disk spectra without additional components. They have obtained satisfactory fits and deduced the disk parameters for a number of sources. They show how the first group sources can be fitted with disk with depleted inner region, while the second group requires more compact structures where the outer region are probably collapsed.

Four different Herbig stars, always selected to show a late spectral type (A to F), and observed with ISOPHOT and LWS, have been studied by Natta et al. (2001). They, applying models of irradiated disk, propose how to solve the puzzle of the excess of near IR emission, with a peak at about $3 \mu \mathrm{m}$, as due to dust evaporation in disks where 
the gas component is optically thin to the stellar radiation, as expected if the accretion rate is very low.

Creech-Eakman et al. (2002) and Chiang et al. (2001) have fitted the LWS spectra of 6 HAEBE (along with 3 T Tauri stars) with a revised version of the Chiang \& Goldreich (1997) model. Only a subset of HAe stars is adequately fitted, showing that the flux at wavelength longward of $55 \mu \mathrm{m}$ arises from the cooler disk interior at large radius, which is heated diffusively by reprocessed radiation from the disk surface. In particular, Chiang et al. (2001) point out how the SED alone does not furnish sufficient information to uniquely constrain the geometry surrounding HAe stars.

A different view is offered by Elia et al. (2004) who compare updated set of observational data spanning 5 orders of magnitude in wavelength and including SWS and LWS spectra, with the model spectra computed for different possible distribution of the circumstellar matter. In their previous model of a spherically symmetric distribution (Pezzuto et al. 1997), cases have been now considered with the circumstellar regions partially evacuated along the polar axis by the action of the stellar wind. The inclusion of the polar cavities indirectly allows geometries in which a small scale disklike flared structure around the central star is present. As a result on a sample of 36 HAEBE, they found that 17 objects are reasonably fitted, eight of which with a pure spherical model, and the remaining nine with the inclusion of the polar cavities. These latter objects are those in common with the Dominik et al. (2003) sample of isolated HAe stars. Such an agreement gives further support to the hypothesis that for low-mass, more evolved HAe stars, the circumstellar geometry can be similar to that found around the T Tauri stars. Moreover they conclude that a dust absorption coefficient typical of larger dust grains has to be associated to these eight cases. This is in agreement with the results by Meeus et al. (2001) and confirms the idea that the grain size is expected to increase with time in circumstellar envelopes as a result of the ongoing growth process activated in high density environments.

Opening a new spectral window longward of $100 \mu \mathrm{m}$, ISO has allowed a classification of Young Stellar Objects (YSOs) more accurate than that provided by IRAS. A new tool has been introduced by Pezzuto et al. (2002) in form of two colours diagram [60-100] vs. [100-170]. The pre-main sequence stars cluster in a definite locus of the plot. The median colours are [60-100] $=0.11 \pm 0.14$ and $[100-170]=-0.01$ \pm 0.12 , corresponding to a colour temperature of about $50 \mathrm{~K}$. This represents a powerful and simple tool to derive indications from the 
future photometric surveys.

The only mid to far IR SEDs of HAEBE constructed with pure photometric ISO data have been presented by Abraham et al. (2000), who examined the ISOPHOT scans obtained with 10 large band filters between 4.8 and $200 \mu \mathrm{m}$ ) across $7 \mathrm{HAEBE}$ (from B0 to A7). The observed SEDs can be described by a power law relationship, but their shapes are inconclusive for ruling out the presence of circumstellar disks or spherically symmetric envelopes. The emission at wavelengths larger than $100 \mu \mathrm{m}$ is dominated by cold objects, unresolved by the $90 \times 90$ $\operatorname{arcsec}^{2}$ pixel of the $200 \mu \mathrm{m}$ camera. These observations support the findings of previous sub-mm maps (Sandell \& Weintraub 1994; Henning et al. 1998) about the existence of dust cores (of $\leq 1^{\prime}$ size) towards many HAEBE. Whether or not these cores coincide with huge massive envelopes (i.e. they are centrosymmetric structures), remains an open question.

\subsection{Dust features and PAH}

Studies of the chemical and mineralogical composition of the dust have received a strong impulse by the ISO advent. It has allowed, for the first time, an unbiased spectroscopical approach to the complete wavelength regime $(3-20 \mu \mathrm{m})$, revealing a large variety in dust properties, from small PAHs (features at 3.3, 6.2, 7.7, 8.6, 11.3, $12.7 \mu \mathrm{m}$ - e.g. Léger \& Puget 1984), nanodiamond (NAN) features (3.4, $3.5 \mu \mathrm{m}$ - van den Kerckhoven et al. 2002), to amorphous (9.7, $18 \mu \mathrm{m})$ and crystalline $(11.3 \mu \mathrm{m})$ silicate dust. Disks around 9 TTS associated with the Cham I dark cloud have been studied by Natta et al. (2000) and Gürtler et al. (1999). All stars show evidence of amorphous silicate emission at $10 \mu \mathrm{m}$, from (sub)-micron sized grains, which is the only prominent feature in those spectra. They discuss how the emission originates in the disk atmosphere, namely the hot layer of the circumstellar disk, which, heated by the stellar radiation, is hotter than the disk midplane and therefore the emission feature can be seen. Chiang et al. (2001) investigate how the SED of the face-on disk around 2 TTS and 3 HAEBE depends on dust properties and grains distributions.

A systematic investigation of ISO SWS and LWS spectra aimed to study the characteristics of the circumstellar dust around a significant sample of HAEBE has been conducted by Meeus et al. (2001). Beside their results on the dust distribution, discussed in the previous section, they also found that the PAH bands are different from the PAH band in the ISM and are only present in HAEBE with a large amount of warm dust. PAH cannot be correlated to any of the stellar parameters 
and are located in an extended region around the disk. Their presence is unrelated to the presence of the silicate bands and these latter are surprisingly laking in some sources.

Acke \& van den Ancker (2004) presented a complete atlas of all the features detected in 46 HAEBE retrieving the spectra (obtained with SWS, PHT-S, CAM-CVF) from the ISO archive. The emission detection rates of PAH, amorphous and crystalline silicates are 57\%, $52 \%$ and $24 \%$, respectively, with some more embedded objects showing silicate in absorption. Following Meeus et al. (2002) and van Boekel et al. (2003), they also provide a classification of HAEBE in two groups based on the ratio between the NIR $(1-5 \mu \mathrm{m})$ and mid-IR $(20-100$ $\mu \mathrm{m})$ luminosity: the systems with stronger mid-IR (relative to their NIR), display significantly more PAH emission than those with weaker mid-IR. This circumstance has been interpreted as an observational support for the disk model by Dullemond et al. (2001) (see Sect. 2.2) in which flaring disks are associated with stronger mid-IR emission, while shielded systems with weaker contributions. Silicates behave differently: since they are expected to be produced mainly in the inner parts of the disk, no strong difference is expected between the two groups.

The composition of the circumstellar dust around four individual HAe has been studied in detail through SWS and LWS spectra. Silicates in the crystalline form (similar to those found in comets), are found in HD100546 (Malfait et al. 1998); C-rich and O-rich components are respectively associated to the warm and cool dust component of HD142527 (Malfait et al. 1999), but no clear correlation exists between dust signatures and stellar parameters. The stars AB Aur and HD163296 (Bouwman et al. 2000; van den Ancker et al. 2000) also show the presence of crystalline silicates and larger $(\sim 1 \mathrm{~mm})$ grain sizes in HD 163296 compared to AB Aur suggests the former as a more evolved system. Bouwman et al. (2000) confirm the poor correlation between the degree of crystallization and the age of the central star and note that crystallization and grain growth are not necessarily coupled. Both these facts signal how the evolution of circumstellar dust in protoplanetary disks happens very rapidly or how such evolution is driven by other factors (than stellar mass and age).

The good quality of the SWS data has allowed Peeters et al. (2002) to derive a striking aspect of the features in the 6-9 $\mu \mathrm{m}$ region, namely their variability: all the features shift in peak position from source to source, show different profile and each seems to be composed of several subfeatures. The HAEBE constitute a small sub-sample among the variety of the investigated objects: their observed variations are discussed in terms of the nature of the carrier and local physical condition, although some aspects remain still unknown. 

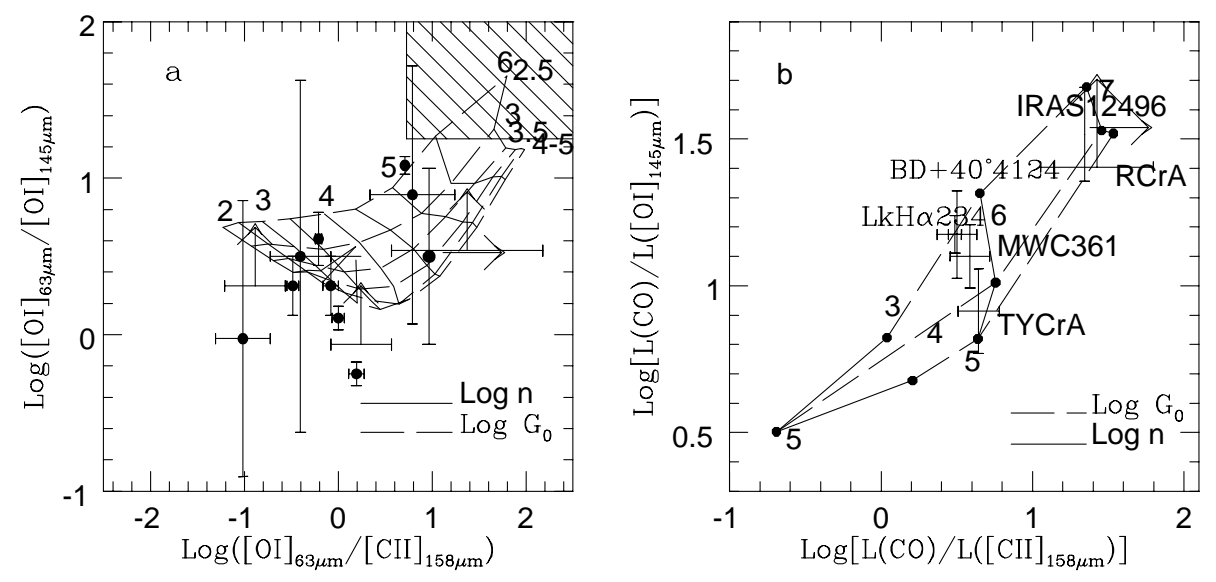

Figure 2. a) Observed line ratios superimposed to PDR models by Kaufman et al. (1999). The hatched area identifies the locus pertaining to the J-shock model predictions. b) Observed ratios between molecular CO lines and fine structure [OI] $145 \mu \mathrm{m}$ and [CII] $158 \mu \mathrm{m}$ superimposed on clumpy PDR models of Burton et al. (1990).

\subsection{EMISSION LINES}

Given the strong underlying continua, combined with the low spectral resolution of the ISO spectrometers (ISOCAM-CVF - ISOPHOT-S SWS - LWS), rather strong emission lines have been detected, often for the first time. The inventory of these lines is given in Table III. The following discussion will address separately the spectral surveys and the studies of individual regions.

\subsubsection{Spectral surveys}

Searches for the $\mathrm{H}_{2}$ pure rotational lines have been performed with SWS. A quite large sample of 7 TTS and 8 HAEBE with reasonable evidence for disks has been investigated by Thi et al. (1999, 2001). They report the detected fluxes (or, in few cases, the $3 \sigma$ upper limits) of the $\mathrm{S}(0)$ and $\mathrm{S}(1)$ lines at 28.2 and $17.0 \mu \mathrm{m}$, respectively. Their conclusion that these lines arise in a warm gas disk component, which constitutes a significative fraction of the entire disk mass, has not been confirmed by the subsequent nondetection of the $\mathrm{S}(1)$ line in some of the same objects (Richter et al. 2002), which conversely suggests that $\mathrm{H}_{2}$ emission could be extended on scales of $5^{\prime \prime}$ or more. However, because of their sensitivity to small amount of warm gas, the mid-IR $\mathrm{H}_{2}$ lines remain crucial for more complete studies with future IR spectrometers. 
In the LWS range the ISO archive has been systematically searched to obtain a complete far IR spectrophotometric survey of HAEBE (Lorenzetti et al. 1999, 2002; Giannini et al. 1999). The investigated sample is constituted by 26 objects all presenting fine structure emission lines from different ions; 6 out of these also show molecular emission in form of $\mathrm{CO}$ and $\mathrm{OH}$ lines usually associated to local density peaks. The [OI] 63, $145 \mu \mathrm{m}$ and [CII] $158 \mu \mathrm{m}$ are the strongest features observed and have been used as a diagnostic of the excitation mechanism and then of the physical conditions. Line ratios and corresponding errors are indicated in Figure 2a. The observed line intensity ratios are superimposed on a grid of photodissociation models (Kaufman et al. 1999). The substantial difference between line intensity predictions of this model and those of other PDR models (e.g. Tielens \& Hollenbach 1985) is the inclusion of an additional heating: the ejection of photoelectrons from PAH and small grains. These latter are essential components of the HAEBE close environments, as demonstrated by many dedicated observations (see e.g. Meeus et al. (2001) and references therein). The extra heating source is essential because for a given value of density $(n)$ and radiation field $\left(G_{o}\right)$, this model predicts increased OI and CII column densities, thus allowing to move at lower $n$ and $G_{o}$ the transition point between CII dominated and OI dominated cooling. The location of the data points in Figure 2a, gives support to the interpretation in favour of phodissociation as dominating mechanism for far IR line excitation (see also Creech-Eakman et al. 2002). The existence, around the HAEBE stars, of a PDR originated by stellar rather than interstellar FUV photons has received further support by the correlation between [CII] $158 \mu \mathrm{m}$ luminosity vs. the bolometric luminosity of the central source (Lorenzetti et al. (1999), their Fig.5).

Alternative models do not seem so much promising: J-shock models (e.g. Hollenbach \& McKee 1989) can be ruled out, since they predict substantially different line ratios whose intersection with the PDR plane is indicated in Figure $2 \mathrm{a}$ as the hatched area at the top right corner. Contributions from non dissociative C-shocks (e.g. Draine et al. 1983) cannot be ruled out, but such mechanism can be considered as the main responsible for the gas excitation just in a narrow region of the parameter space (shock speed, magnetic field, pre-shock density).

The molecular lines are usually weak (Giannini et al. 1999), but the authors do not believe the lack of detection is due only to an instrumental sensitivity limit, but it is also related to an intrinsic property of the circumstellar environment, namely the existence or not of some compact density peaks near the source where the column density is expected to substantially increase. For all sources the molecular emis- 
sion has been consistently fitted with a Large Velocity Gradient (LVG) model and it results originated in a warm $(\mathrm{T} \geq 200 \mathrm{~K})$ and dense $(n$ $\sim 10^{5} \mathrm{~cm}^{-3}$ ) gas located in very compact regions having diameters of few hundreds of AU. By comparing the observed cooling ratios $(\mathrm{L}(\mathrm{CO}) / \mathrm{L}([\mathrm{OI}]), \mathrm{L}(\mathrm{CO}) / \mathrm{L}([\mathrm{CII}]))$ with model predictions Burton et al. 1990, see Figure 2b) the photondominated mechanism in high density or clumpy environments appears to be the likely source of excitation, while shocks can reasonably ruled out because of both the absence of any water vapour lines and the presence of $\mathrm{OH}$ lines, in contrast with predictions of molecular emission.

Fine structure lines from ionized species [OIII], [NII] are detected in those HAEBE having a spectral type B0 or earlier (see Table III, and these individual cases will be discussed in the next section.

Finally, studies have been presented (Lorenzetti et al. 2002) to derive some general trend to be compared with similar behaviours from classes of younger objects. The far IR luminosity $\left(\mathrm{L}_{F I R}\right)$ as due to all line emission contributions (fine structure line emission and total molecular cooling) is plotted in Figure 3 as a function of the bolometric luminosity of the central object. The straight line, whose equation is given in the upper part of the Figure, represents the best linear fit to the points: the intercept on the $y$-axis is located at a $\mathrm{L}_{F I R}$ value of about $10^{-4}$. A similar plot for the younger Class 0 objects (Nisini et al. 2002, here depicted as triangles) indicates $\mathrm{L}_{F I R} \simeq 10^{-2}$ as the intercept value. An intermediate value of $10^{-3}$ is attributable to the Class I objects (open circles), which are expected to be somehow younger than HAEBE and approaching to their evolutionary stage. Although completely different mechanisms are invoked to account for the behaviour of the HAEBE and of the other objects (photodissociation vs. shock excitation), it

is worthwhile noting how $\mathrm{L}_{F I R}$ is a decreasing fraction of $\mathrm{L}_{b o l}$ while the evolution goes on. The conversion of the bolometric luminosity of the central object into far IR line cooling in the circumstellar envelope occurs at progressively lesser efficiency while time elapses. Therefore ISO has allowed to extend to the far IR gas cooling the well consolidated statement according to which the relevance of the circumstellar vs. stellar properties diminishes during the pre-stellar evolution.

\subsubsection{Emission lines of individual objects}

Here, account is given for those TTS, HAEBE or nearby regions that have been individually investigated with the ISO spectrometers, through the analysis of emission lines different from those presented in the previous section, or through different techniques (e.g. spectroscopic 


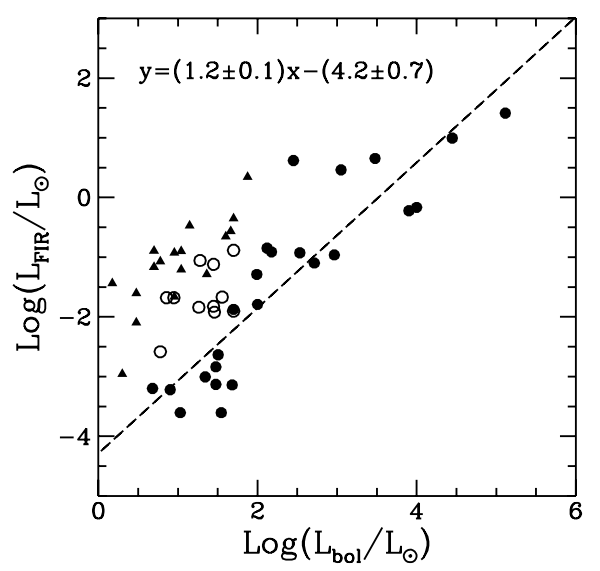

Figure 3. Far IR lines luminosity vs. bolometric luminosity of the central star. The equation representing the best linear fit to the points (dashed line) is given in the upper part. HAEBE are represented as filled circles, while younger Class 0 and Class I objects (see text) are indicated with triangles and open circles, respectively.

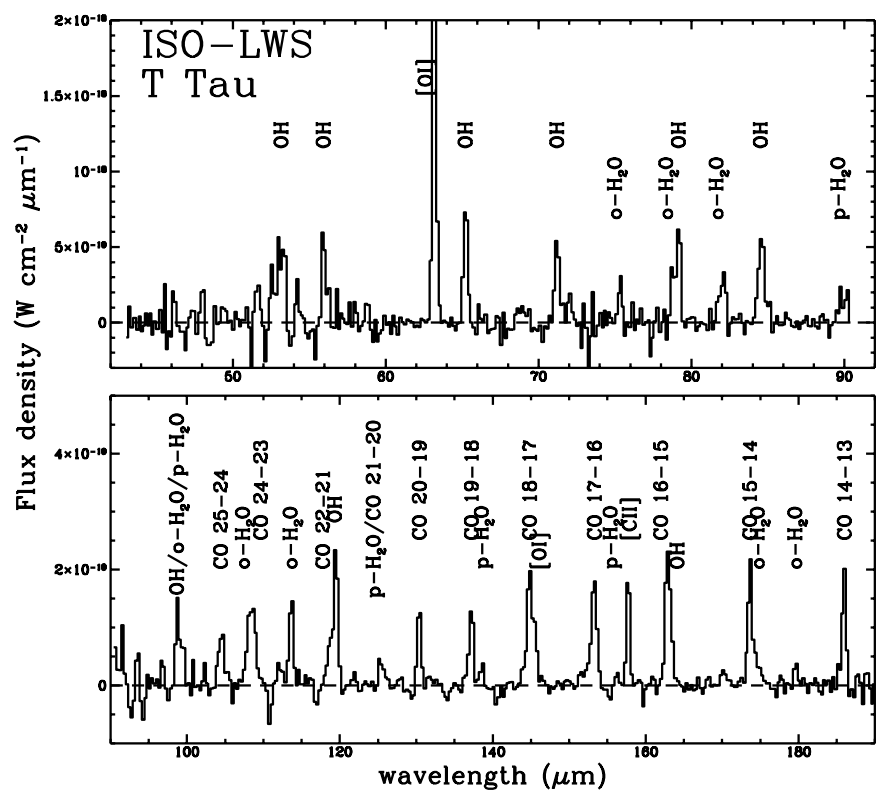

Figure 4. The ISO-LWS emission line spectrum of T Tau, from which the continuum has been subtracted. 
mapping).

T Tauri - This binary system presents the richest SWS (van den Ancker et al. 1999) and LWS (Spinoglio et al. 2000) spectrum (see Figure 4), which signals the presence of different excitation mechanisms: a partially ionized wind is likely responsible for both the HI recombination lines and FIR fine structure lines through the onset of shocks. These latter are also traced by a low temperature $(T \approx 400$ $\mathrm{K})$ component of the pure rotational and rovibrational emission from $\mathrm{H}_{2}$. The role of the UV field is evidenced by both a higher temperature component $\left(\mathrm{T} \approx 1500 \mathrm{~K}\right.$ ) of $\mathrm{H}_{2}$ and the overabundance of $\mathrm{OH}$ (more than a factor of 10). Most of the observed FIR molecular lines (high $\mathrm{J}$ $(\mathrm{J}=14-25) \mathrm{CO}$, para- and ortho- $\left.\mathrm{H}_{2} \mathrm{O}, \mathrm{OH}\right)$ can be attribute to a very compact region $(300-400 \mathrm{AU})$ with $\mathrm{T} \sim 300-900 \mathrm{~K}$ and $\mathrm{n}_{H_{2}} \sim 10^{5-6}$ $\mathrm{cm}^{-3}$

CoD-42 ${ }^{\circ} 11721$ and MWC1080 - These two HAEBE (with Sp.T B0) have been studied by Benedettini et al. (1998). Their SWS spectra show hydrogen recombination lines of the Brackett, Pfund and Humphreys series (see Table III). The observed line decrements in each spectral series are consistent with emission from ionized winds, as expected for early type stars. Mass loss rates and reconsidered distance values have been derived along with the presence of ionization bounded compact regions of a few tens of stellar radii. In the case of CoD$42^{\circ} 11721$, a more diluted ionized region (with electron density $\mathrm{n}_{e} \approx 5$ $10^{2} \mathrm{~cm}^{-3}$ ), external to the optically thick HII region, is traced by the fine structure line ratios [SIII] 33/19 and [OIII] 88/52 (Lorenzetti et al. 1999). ISO observations indicate that density gradients, more than abundance gradients, are responsible for the observed line ratios, thus contrasting a previous interpretation in favour of an evolved nature of this star (Hutseméker \& van Drom 1990).

MWC297 - The same model of ionized wind has been applied to the plenty (more than 20) recombination lines in the SWS range (Benedettini et al. 2001), providing consistent determinations of the distance $(280 \mathrm{pc})$, the visual extinction $(7.5 \mathrm{mag})$, the mass loss rate $\left(910^{7} \mathrm{M}_{\odot} \mathrm{yr}^{-1}\right)$ and the size of the emitting ionized region (30 stellar radii).

$\mathrm{BD}+\mathbf{4 0} \mathbf{4 1 2 4}^{\circ}$ - It belongs to a group together with the HAe $\mathrm{LkH} \alpha$ 224 and the embedded source $\mathrm{LkH} \alpha 225$. In the first SWS study of this complex (Wesselius et al. 1996) the detection of $\mathrm{H}_{2}$ pure rotational lines is reported. The analysis indicates excitation temperatures of about 500 
$\mathrm{K}$ and a very weak $\mathrm{C}$-type shock wave driven into the cloud by stellar outflows as the most likely mechanism for the $\mathrm{H}_{2}$ excitation. More recently, van den Ancker et al. (2000) presented the spectra of both SWS and LWS of the three member of the group, where several contributions to the emission (pure rotational lines of $\mathrm{H}_{2}$, ro-vibrational transition of $\mathrm{CO}$, PAHs, HI recombination lines and IR fine structure lines) were detected. Again, the data suggest that the HI lines of $\mathrm{BD}+40^{\circ} 4124$ arise in either a dense wind or a circumstellar disk, while a low density $\left(\approx 10^{2} \mathrm{~cm}^{-3}\right)$ HII region combined with a clumpy PDR is responsible for the FIR fine structure lines. Lines from non-dissociative shock are observed in the lines of sight of $\mathrm{LkH} \alpha 224$ and $\mathrm{LkH} \alpha 225$ produced by a slow outflow driven by the latter, toward which also a dissociative shock is recognized, presumably located closer to this exciting source.

NGC7023 - It is a reflection nebulosity hosting the HAEBE star HD200775 and a very close (50") PDR that has been studied by Fuente et al. (1999), who provide a possible explanation (the advancing photodissociation front) for the nonequilibrium ortho-to-para ratio of 1.5-2 they found at variance with other ISO $\mathrm{H}_{2}$ observations toward several Galactic regions (see Habart, this issue). A larger portion of the same region, including also HD200775, has been investigated later (Fuente et al. 2000) and strong emission of the [SiII] $34.8 \mu \mathrm{m}$ line has been detected towards the star, filling a ring constituted by the PDR tracers ([OI[, [CII]).This morphology can be only explained as arising in the diffuse gas filling the cavity around the star and its HII region. Indeed only up to $\mathrm{A}_{V} \sim 2$ a significant fraction of Si remains in the gas phase, while for larger $\mathrm{A}_{V}$ values $\mathrm{Si}$ is strongly depleted.

NGC7129 - LWS has also been used to provide a FIR spectroscopic map of this complex star forming region (Lorenzetti et al. 1999) where two HAEBE are located, namely $\mathrm{BD}+65^{\circ} 1637$ and $\mathrm{LkH} \alpha 234$. [OI] $63,145 \mu \mathrm{m}$ and [CII] $158 \mu \mathrm{m}$ lines are detected everywhere in the mapped region and their intensity ratios are consistent with the model prediction of a PDR. The spatial distribution of the emission features indicates the presence of a peak which not coincide with the position of $\mathrm{LkH} \alpha 234$, but it seems more likely associated with the other HAEBE. This view is supported by the distribution of the ${ }^{13} \mathrm{CO}$ emission obtained, at a comparable scale, by Fuente et al. (1998), where the cavity excavated by $\mathrm{BD}+65^{\circ} 1637$ is clearly evident. 


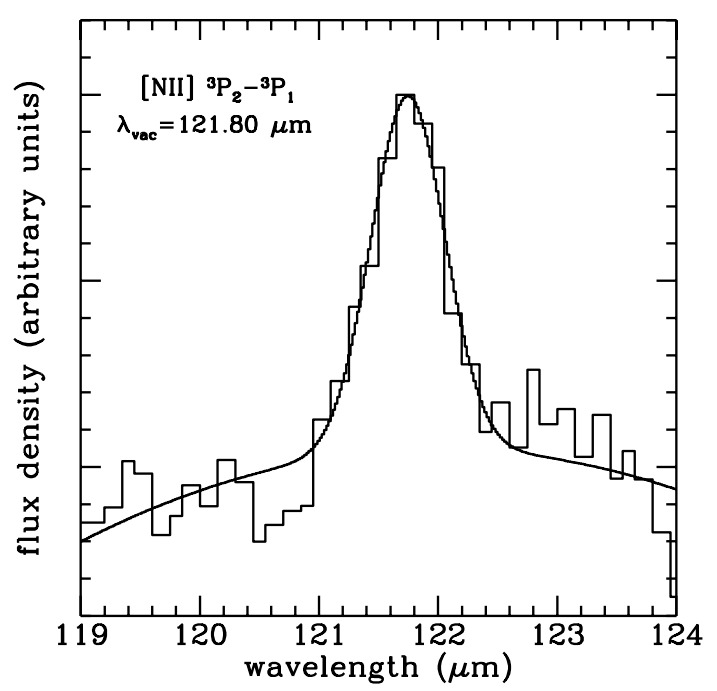

Figure 5. Mean spectrum in the range 119-124 $\mu \mathrm{m}$ obtained by averaging all the individual FU Ori spectra. The superposed formal fit provides a detection at $\mathrm{S} / \mathrm{N}>8$, with a width equal to the instrumental one, and centered at $121.75 \mu \mathrm{m}$.

\section{FU Ori systems}

An other sample, interesting for having a complete picture of the premain sequence evolution, is represented by the FU Orionis stars: low luminosity objects which undergo a sudden increase in brightness. The widely accepted explanation of the FU Orionis phenomenon is a large increase in the accretion rate through a circumstellar disk surrounding a low luminosity young star (Hartmann \& Kenyon 1985). These events make the disk self luminous, with a luminosity which largely exceeds that of the central star (for more details see the review papers by Hartmann et al. 1993; Hartmann \& Kenyon; and references therein). The observations obtained with the ISO instrumentation are reported in the last three columns of Table I. These data essentially consist of the LWS spectra presented by Lorenzetti et al. (2002). In addition to [OI] $63 \mu \mathrm{m}$ and the [CII] $158 \mu \mathrm{m}$ lines, that are commonly observed in all spectra, the observational novelty is the presence in most of the sources of the transition of ionized nitrogen [NII] $122 \mu \mathrm{m}$, which is not detected in other objects in a similar evolutionary phase. This line probes low ionization and low density material $\left(\mathrm{n}_{e}<510^{2} \mathrm{~cm}^{-3}\right)$ not easily traced by other lines. No higher ionization stages of other elements are detected. It is worthwhile to mention that the [NII] detections in FU Ori are close to the limit of the observing capabilities and, as 
such, they could be arguable; however the circumstance to find this line practically at all the pointings on FU Ori objects, surely increases the statistical confidence in ascribing this property to this specific class. To emphasize this point in Figure 5 a sort of mean spectrum is reported: the result demonstrates that [NII] line is indeed real.

A possible interpretation of the observed FIR spectra is based on the simultaneous presence of two components: (i) a well localized J-shock, responsible for the $[\mathrm{OI}]$ emission, and (ii) an extended low density ionized medium, produced by the UV photons from the disk boundary layer $\left(\mathrm{T}_{\text {eff }} \sim 310^{4} \mathrm{~K}\right.$ ), responsible for the [NII] and [CII] emission. Infact, in FU Ori systems, the central star (F-G type) cannot provide the requisite ionising photons $\left(\mathrm{G}_{o} \approx 0.1\right.$ at $\left.0.1 \mathrm{pc}\right)$.

\section{Future perspectives}

The FIR spectroscopical surveys presented here have clarified many aspects of the interaction between pre-main sequence objects and their close environment, and have originated new questions which can be hopefully answered by using the forthcoming facilities. Some relevant aspects, addressed in this review, are summarized in the following:

- ISO instrumentation has evidenced for the first time the multiplicity of phenomena (outflows, shocks, disk emission, fluorescence) occurring in complex systems or in young binary objects (such as $\mathrm{T}$ Tau), but, due to the large beam size, one is often unable to specify which is the origin of the different manifestations.

- Surveys of $\mathrm{H}_{2}$ pure rotational lines (in the 7-28 $\mu \mathrm{m}$ range) revealed themselves as crucial to probe small amounts of warm gas (maybe disks/planets) around young embedded objects, but enough spatial resolution (better than 1 arcsec) is required to impose compelling constrains.

- The ISO-LWS mapping capability is enough to derive (from [OI] and [CII] line ratios) the gross distribution of $G_{o}$ and density. A better spatial resolution of $\sim 10 \operatorname{arcsec}$ is in order to investigate the internal structure of the detected PDR's by tracing the transition zones $\mathrm{C}^{+} / \mathrm{C} / \mathrm{CO}$, thus clarifying whether or not clumpiness is an important ingredient. 
- The observed ratio [OI] $63 \mu \mathrm{m} /[\mathrm{OI}] 145 \mu \mathrm{m}$ is always lesser than predicted from both standard PDR and J- C-shocks models. To find a satisfactory agreement with the observed data, we pushed the PDR models toward the lowest [OI] 63/145 values. Studying whether this large scale behaviour stems from properties intrinsic to the emitting gas or results from averaging different emission vs. absorption components, deserves a spatial resolution much better (by $\sim$ an order of magnitude) than that of ISO-LWS.

- Molecular emission in HAEBE has been detected on a few sources, coming from very compact $(\leq 10$ arcsec) regions. A systematic study carried out with a better sensitivity and spatial resolution could both give a definitive answer to the size of the emitting regions and significantly increase the detection rate of molecular emission (which is, at the moment, quite low).

- ISO missed the fine structure transition of [NII] at $205 \mu \mathrm{m}$. The diagnostic diagram which uses the [NII] line pair $(122 / 205 \mu \mathrm{m}$, see e.g. Rubin et al. 1994), would provide the electron density in the $\mathrm{N}^{+}$ volume and the fractional ionization in the environment around FU Ori objects. This will allow to verify the hypothesis about the [NII] excitation mechanism in that class. Moreover, at higher spectral resolution, the [NII] $205 \mu \mathrm{m}$ line (expected to be brighter than the $122 \mu \mathrm{m}$ one) can be resolved, allowing a better constrain on the origin of this line.

\section{References}

Acke B., van den Ancker M. 2004 A\&A submitted

Abraham P., Leinert C., Burkert A., Henning T., Lemke D. 2000 A\&A 354, 965

Benedettini M., Nisini B., Giannini T., et al. 1998 A\&A 339, 159

Benedettini M., Pezzuto S., Giannini T., Lorenzetti D., Nisini B. 2001 A\&A 379, 557

Blommaert, J., Siebenmorgen, R., Coulais, A. et al. 2003, 'The ISO Handbook, Volume II: CAM - The ISO Camera', ESA SP-1262

Bouwman J., de Koeter A., Dominik C., Waters L.B.F.M. 2003 A\&A 401, 577

Bouwman J., de Koeter A., van den Ancker M.E., Waters L.B.F.M. 2000 A\&A 360, 213

Bouwman J., Meeus G., de Koeter A., Hony S., Dominik C., Waters L.B.F.M. 2001 A\&A 375, 950

Burton M.G., Hollenbach D., Tielens A.G.G. 1990 ApJ 365, 620

Cesarsky, C.J. et al. 1996, A\&A 315, L32

Chiang E.I., Goldreich P. 1997 ApJ 490, 368

Chiang E.I., Joung M.K., Creech-Eakman M.J., Qi C., Kessler J.E. 2001 ApJ 547, 1077

Clegg, P.E. et al. 1996, A\&A 315, L38 
Creech-Eakman M.J., Chiang E.I., Joung R.M.K., Blake G.A., van Dishoeck E.F. 2002 A\&A 385, 546

de Graauw, T. et al. 1996, A\&A 315, L49

Dominik C., Dullemond C.P., Waters L.B.F.M., Walch S. 2003 A\&A 398, 607

Draine B.T., Roberge W.G., Dalgarno A. 1983 ApJ 264, 485

Dullemond C.P., Dominik C., Natta A. 2001 ApJ 560, 957

Elia D., Strafella F., Campeggio L., Giannini T., Lorenzetti D., Nisini B. 2004 ApJ 601,1000

Fuente A., Martiín-Pintado J., Bachiller R. Neri R., Palla F. 1998 A\&A 334, 253

Fuente A., Martiín-Pintado J., Rodríguez-Fernández N.J., Chernicharo J., Gerin M. 2000 A\&A 354, 1053

Fuente A., Martiín-Pintado J., Rodríguez-Fernández N.J., Rodríguez-Franco A., de Vicente P., Kunze D. 1999 ApJ 518, L45

Giannini T., Lorenzetti D., Tommasi E., et al. 1999 A\&A 346, 617

Gry, C., Swinyard, B., Harwood, A. et al. 2003, 'The ISO Handbook, Volume III: LWS - The Long Wavelength Spectrometer', ESA SP-1262

Gürtler J., Schreyer K., Henning T., Lemke D., Pfau W. 1999 A\&A 346, 205

Hartmann L.W., Kenyon S.J. 1985 Ap.J. 299, 462

Hartmann L.W., Kenyon S.J. 1996 Ann.Rev.Astr.Astrophys. 34, 207

Hartmann L.W., Kenyon S.J., Hartigan P. 1993 Protostars and Planets III, Eds. E.H.Levy, J.I.Lunine, Univ. Arizona Press p.497

Henning T., Burkert A., Launhardt R., Leinert C., Stecklum B. 1998 A\&A 291, 546

Hollenbach D., McKee C.F. 1989 ApJ 342, 306

Hutseméker D., van Drom E. 1990 A\&A 238, 134

Kaufman M.J., Wolfire M.G., Hollenbach D.J., Luhman M.L. 1999 ApJ 527, 795

Kessler, M.F. et al. 1996, A\&A 315, L27

Kessler, M.F., Mller, T.G., Leech, K. et al. 2003, 'The ISO Handbook, Volume I: ISO - Mission \& Satellite Overview', ESA SP-1262

Laureijs, R.J., Klaas, U., Richards, P.J. et al. 2003, 'The ISO Handbook, Volume IV: PHT - The Imaging Photo-Polarimeter', ESA SP-1262

Leech, K., Kester, D., Shipman, R. et al. 2003 ESA SP-1262

Léger A., Puget J.L. 1984 A\&A 137, L5

Lemke, D. et al. 1996, A\&A 315, L64

Lorenzetti D., Giannini T., Nisini B., et al. 2000 A\&A 357, 1035

Lorenzetti D., Giannini T., Nisini B., et al. 2002 A\&A 395, 637

Lorenzetti D., Tommasi E., Giannini T., et al. 1999 A\&A 346, 604

Malfait K., Waelkens C., Bouwman J., de Koter A., Waters L.B.F.M. 1999 A\&A 345,181

Malfait K., Waelkens C., Waters L.B.F.M., Vandenbussche B., Huygen E., de Graauw M.S. 1998 A\&A 332, L25

Meeus G., Waters L.B.F.M., Bouwman J., van den Ancker M.E., Waelkens C., Malfait K. 2001 A\&A 365, 476

Natta A., Meyer M.R. Beckwith S.V.W. 2000 ApJ 534, 838

Natta A., Pusti T., Neri R., Wooden D., Grinin V.P., Mannings V. 2001 A\&A 371, 186

Nisini B., Giannini T., Lorenzetti D. 2002 ApJ 574, 246

Nisini B., Lorenzetti D., Cohen M., et al. 1996 A\&A 315, L321

Peeters E., Hony S., van Kerckhoven C. et al. 2002 A\&A 390, 1089

Pezzuto S., Grillo F., Benedettini M., et al. 2002 MNRAS 330, 1034

Pezzuto S., Strafella F., Lorenzetti D., 1997 ApJ 485, 290

Richter M.J., Jaffe D.T., Blake G.A., Lacy J.H. 2002 ApJ 572, L161 
Rubin R.H., Simpson J.P., Lord S.D. 1994 ApJ 420, 772

Sandell G., Weintraub D.A. 1994 A\&A 292, L1

Siebenmorgen R., Natta A., Kruegel E., Prusti T. 1998 A\&A 339, 164

Siebenmorgen R., Prusti T., Natta A., Müller T.G. 2000 ApJ 561, 1074

Spinoglio L., Giannini T., Nisini B. et al., 2000 A\&A 353, 1055

Thi W.F., van Dishoeck E.F., Blake G.A., van Zadelhoff G.-J., Hogerheijde M.R. 1999 ApJ 521, L63

Thi W.F., van Dishoeck E.F., Blake G.A., et al. 2001 A\&A 385, 546

Tielens A.G.G.M., Hollenbach D. 1985 ApJ 291, 722

van Boekel R., Waters L.B.F.M., Dominik C. et al. 2003 A\&A 400, L21

van den Ancker M.E., Bouwman J., Wesselius P.R., Waters L.B.F.M., Dougherty S.M., van Dishoeck E.F. 2000a A\&A 357, 325

van den Ancker M.E., Wesselius P.R., Tielens A.G.G.M. 2000b A\&A 355, 194

van den Ancker M.E., Wesselius P.R., Tielens A.G.G.M., van Dishoeck E.F., Spinoglio L. 1999 A\&A 348, 877

van den Kerckhoven C., Tielens A.G.G.M., Waelkens C. 2002 A\&A 384, 568

van Dishoeck E.F. 2004 ARA\&A 42, 119

Wesselius P.R., van den Ancker M.E., Young E.T, et al. 1996 A\&A 315, L197 